Documentation et bibliothèques

DOCUMENTATION BIBLIOTHËQUES

\title{
Fides... 50 ans de présence à notre culture
}

FIDES... A half Century of our Culture

FIDES... una presencia de 50 años en nuestra cultura

\section{Jean-Rémi Brault}

Volume 34, numéro 3, juillet-septembre 1988

URI : https://id.erudit.org/iderudit/1052485ar

DOI : https://doi.org/10.7202/1052485ar

Aller au sommaire du numéro

\section{Éditeur(s)}

Association pour l'avancement des sciences et des techniques de la documentation (ASTED)

ISSN

0315-2340 (imprimé)

2291-8949 (numérique)

Découvrir la revue

Citer cet article

Brault, J.-R. (1988). Fides... 50 ans de présence à notre culture. Documentation et bibliothèques, 34(3), 93-97. https://doi.org/10.7202/1052485ar

\section{Résumé de l'article}

Après une brève rétrospective de l'édition au Québec, l'auteur décrit le répertoire et les diverses collections publiées pendant cinquante ans aux Éditions Fides dont la politique éditoriale est basée sur la notion de l’humanisme intégral élaborée par Jacques Maritain.
Tous droits réservés (C Association pour l'avancement des sciences et des techniques de la documentation (ASTED), 1988
Ce document est protégé par la loi sur le droit d'auteur. L'utilisation des services d'Érudit (y compris la reproduction) est assujettie à sa politique d'utilisation que vous pouvez consulter en ligne.

https://apropos.erudit.org/fr/usagers/politique-dutilisation/ 


\title{
Fides... 50 ans de présence à notre culture
}

\author{
Jean-Rémi Brault \\ Archives nationales du Québec \\ Montréal
}

Après une brève rétrospective de l'édition au Québec, l'auteur décrit le répertoire et les diverses collections publiées pendant cinquante ans aux Éditions Fides dont la politique éditoriale est basée sur la notion de I'humanisme intégral élaborée par Jacques Maritain.

\section{FIDES... A half Century of our Culture}

Following a short historical outline of publishing in Québec, the author describes the books and series published by the Editions Fides over a 50year period. The editorial policy of the Éditions Fides is inspired by the concept of integrated humanism as developped by Jacques Maritain.

\section{FIDES... una presencia de 50 años en nuestra cultura}

Después de un breve resumen histórico de la edición en la provincia de Québec, el autor describe el repertorio y las distintas colecciones que se publicaron a lo largo de los últimos cincuenta años en la casa editorial Fides cuya política de publicación se basa sobre la noción del humanismo integral elaborado por Jacques Maritain.
La décennie qui précède la deuxième guerre mondiale, faut-il le rappeler, a été marquée, surtout sur le plan économique, par des années fort difficiles. Depuis 1929, l'année du «krach» économique, de triste mémoire, la situation du chômage atteignait un niveau inquiétant. La misère avait tendance à se généraliser et à envahir toutes les classes de notre société.

Aussi, la recherche du «primo vivere» n'incitait pas ceux qu'on appelait à l'époque les Canadiensfrançais à rechercher les plaisirs de la vie intellectuelle. Même la bourgeoisie, peut-être moins durement frappée par la misère et ses séquelles, était peu tourmentée par la vie intellectuelle. Cette petite société de francophones du Québec, en fait, luttait d'abord pour sa survie matérielle et, de façon sans doute moins consciente, pour sa survie sociale.

Pourtant, une poignée de courageux éditeurs maintenaient une activité éditoriale étonnante tant par sa qualité que par sa quantité. Même si le souvenir de ces valeureux s'est plus ou moins estompé dans le mémorial de leurs compatriotes, l'objectivité nous impose de reconnaître le caractère essentiel de leur contribution à l'évolution intellectuelle de leurs concitoyens.

\section{L'édition au Québec: un peu d'histoire}

Sans dresser une chronologie complète que, espérons-nous, une éventuelle histoire de l'édition au Québec permettrait d'élaborer, il convient de rappeler les faits suivants. Fondée en 1842 par Charles-Odilon Beauchemin, la Librairie Beauchemin poursuit toujours ses activités durant la décennie qui nous occupe. Malgré le nom officiel qu'elle porte, elle publie de nombreux ouvrages qui exercent une influence décisive, particulièrement dans le domaine de l'enseignement. De même, les Éditions Granger Frères, fondées en 1885 par l'homme d'affaires Flavien Granger, continuent de publier des ouvrages fort répandus, dont un bon nombre de manuels scolaires. De leur côté, les Jésuites ont créé, en 1920, les Éditions Bellarmin qui se spécialisent non seulement dans le livre à caractère religieux mais aussi dans la publication d'ouvrages qui assurent la diffusion de la doctrine sociale de l'Église. Puis, en 1922, les Éditions Édouard Garand se lancent, avec un certain succès, dans l'édition populaire accessible pour un prix modique. Et en 1926, Albert Lévesque, cet infatigable nationaliste, fonde la maison d'édition qui portera son nom et qui exercera une influence exceptionnelle sur l'histoire de l'édition 
au Québec et sur l'histoire de la vie intellectuelle et culturelle tout entière. Qu'il suffise de rappeler qu'en 1936, il avait déjà publié plus de deux cent cinquante ouvrages d'auteurs québécois aussi importants que Lionel Groulx, Jean Bruchési, Marie-Claire Daveluy, Édouard Montpetit, Louis Dantin, Harry Bernard, Robert Choquette, Alfred Desrochers, Jovette Bernier, Rex Desmarchais, François Hertel, pour n'en mentionner que quelques-uns.

Puis, durant la décennie 1930,. c'est d'abord Albert Pelletier qui fonde les Éditions du Totem, en 1933. Et l'année suivante, sont créées les Éditions du Zodiaque que dirigera le prolifique Eugène Achard qui aurait suffi, presque à lui seul, à alimenter une maison d'édition. Et encore en 1936, les Éditions Bélisle qui se spécialisent surtout dans le livre technique. Puis vinrent, l'année suivante, les Éditions Fides.

\section{Une politique éditoriale}

On peut dire que, dès le point de départ, les Éditions Fides se sont identifiées à l'Action catholique canadienne, et plus spécifiquement à la Jeunesse étudiante catholique (JEC). Cette maison d'édition est même née grâce à l'utilisation de la méthodologie spécifique aux divers mouvements d'Action catholique.

Rappelons que cette méthodologie consistait à pratiquer le «voir, juger, agir». En clair, cela signifie qu'avant de prendre une décision, avant d'«agir», il convient d'examiner la situation, de l'ausculter, d'enquêter, puis de porter un jugement qui conduira normalement à poser des gestes.

C'est ce qui s'est produit en 1936. La Jeunesse étudiante catholique procède à une vaste enquête sur le problème de la lecture chez les jeunes. C'est l'étape du "voir». Déjà, dans divers milieux, chez les éducateurs, les parents, les militants, on intuitionnait que «les étudiants lisaient peu, lisaient mal et que ce serait important pour la JEC de les aider à s'améliorer au point de vue culturel». C'est le Père Paul-Aimé Martin, c.s.c., qui le mentionne dans une entrevue donnée sur les ondes de la Société Radio-Canada.

Le résultat de cette enquête est éloquent. II consolerait même les personnes qui se désolent de la situation actuelle. Les étudiants de cette époque lisaient peu; et quand ils lisaient, ils lisaient mal; ils retenaient peu; ils ne savaient ni prendre des notes ni tirer un quelconque profit de leurs rares lectures. La réflexion sur les résultats de cette enquête, le «juger», pourrait conduire à culpabiliser les parents comme les éducateurs, à accuser les jeunes de toutes les faiblesses possibles sinon à leur prédire un avenir plutôt incertain.

Plus positivement, plus concrètement, le Père Martin, encore étudiant en théologie, avec l'accord des dirigeants des mouvements de I'Action catholique et avec celui de ses supérieurs, décide de proposer un premier élément de solution à cette situation. II crée un périodique qui s'appellera Mes fiches.

\section{Voici ce qu'il raconte:}

Avec des confrères, avec l'appui des supérieurs, de la JEC, j'ai lancé le 1er mars 1937, la revue Mes fiches. Cette revue se présentait sous forme d'un cartable, si on peut dire, dans lequel se trouvaient seize feuilles séparées contenant chacune un résumé d'un article de revue, de journal ou d'un chapitre d'un volume. Et chaque titre comportait la cote de la classification décimale, de telle sorte qu'on pouvait la classer dans un fichier.

La revue a eu du succès immédiatement. Le premier numéro avait été tiré à 2000 exemplaires, mais au bout d'un mois c'était épuisé, et dès lors le tirage s'est établi à 10000 exemplaires. Une grande partie était destinée à des abonnés individuels, mais la majorité du tirage s'en allait dans les collèges qui en achetaient des quantités comme cinquante ou cent numéros.

Le succès nous a surpris, mais en somme c'était un signe que cette revue répondait à un besoin très réel. La revue, pendant les années qui ont suivi, publiait aussi des brochures et devenait lentement une maison d'édition mais aussi toujours au nom de Mes fiches.

C'est en 1940 que, officiellement, cette institution adopte le nom de Fides. Et dès le début, la nouvelle maison d'édition épouse les préoccupations de l'humanisme intégral, particulièrement diffusé par le philosophe français Jacques Maritain. Rappelons que, pour cet auteur lui-même, I'humanisme intégral

tend essentiellement à rendre l'homme plus vraiment humain, et à manifester sa grandeur originelle en le faisant participer à tout ce qui peut l'enrichir dans la nature et dans l'histoire [«en concentrant le monde en l'homme», comme disait à peu près Scheler, et en «dilatant I'homme au monde"]; il demande tout à la fois que I'homme développe les virtualités contenues en lui, ses forces créatrices et la vie de la raison, et travaille à faire des forces du monde physique des instruments de sa liberté. 
Ainsi entendu, l'humanisme est inséparable de la civilisation ou de la culture ces deux mots étant eux-mêmes pris pour synonymes

Toute la politique éditoriale de Fides est contenue dans cette notion de l'humanisme intégral

élaborée par Jacques Maritain. Et, bien sûr, I'Action catholique en constitue le point de départ, le volet essentiel et primordial, sans négliger la formation et le développement complets des individus. Au contraire, puisque, selon l'auteur, l'excellence de ce travail en action catholique exige «la compétence et les armes voulues».

II ne faudra donc pas s'étonner que, dès le début de ses activités, Fides publie cinquante-six ouvrages, dont onze ne comportent aucun caractère religieux. Signalons, entre autres, Le Saguenay historique et Le Saguenay pittoresque de Elphège Brassard, des biographies de Hitler et de Roosevelt [la deuxième grande guerre fait rage], une Initiation à la géologie [qui connaîtra un succès considérable et plusieurs éditions et réimpressions], par Laverdière et Morin, ainsi que la Notion de milieu, qui constitue le résultat d'une enquête sociale fort importante.

Seules les personnes qui ignoreront ou auront oublié cette politique éditoriale initiale, dûment adoptée par les Éditions Fides dès le début de ses activités, s'étonneront que cette maison d'édition, à direction religieuse, publie des ouvrages qui ne comportent aucune connotation religieuse. Il est intéressant de vérifier et de constater que cette politique a été appliquée régulièrement tout au long de ces cinquante années.

Par la même occasion, nous observons l'importance des oeuvres de cette maison d'édition dans I'histoire culturelle du Québec. À une époque où les universités québécoises ne se sont pas encore dotées de «presses universitaires», les Éditions Fides ont rempli cette place, ont joué ce rôle, sans le dire, sans en parler, en acceptant de publier quelquefois des ouvrages savants, avec une efficacité d'autant plus étonnante qu'elles n'en avaient pas les moyens.

Graduellement, et même très rapidement, les responsables de cette maison ont voulu appliquer et intégrer leur notion d'humanisme intégral à l'ensemble de la collectivité francophone canadienne, et non pas seulement aux jeunes ou aux militants de l'Action catholique. Offrir des ouvrages de bonne qualité dans toutes les disciplines du savoir humain, faire connaître et diffuser les meilleurs ouvrages d'auteurs français et canadiens, rendre accessibles à des prix concurrentiels des oeuvres qui, autrement, n'auraient été connues que par le truchement d'éditeurs européens, c'était vivre activement la notion d'humanisme intégral.

C'est pourquoi la politique éditoriale de la maison imposait de ne pas se limiter aux ouvrages à caractère religieux même si cette maison d'édition relevait directement d'une communauté religieuse. Nous avons voulu vérifier la véracité de cette assertion. Nous avons fait un inventaire des publications des Éditions Fides, pour les cinquante premières années, soit de 1937 à 1987 . Durant cette période, 1882 volumes ont été publiés. De ce nombre:

554 appartiennent à la catégorie «livres religieux";

387 sont des ouvrages de littérature;

297 peuvent être classés «livres de jeunesse»;

194 sont des ouvrages historiques;

117 sont des biographies;

42 peuvent être classés en sciences pures ou appliquées;

291 ouvrages appartiennent à diverses disciplines profanes.

Quelques remarques s'imposent pour bien situer ces statistiques dans leur juste perspective.

\section{Le répertoire}

Les ouvrages religieux répertoriés couvrent tous les secteurs de cette discipline. On y trouve aussi bien les encycliques pontificales et les missels que des ouvrages d'exégèse biblique, de liturgie, de théologie ou de réflexion spirituelle. On ne s'étonnera pas que les livres religieux comptent pour le tiers des publications des Éditions Fides. D'aucuns pourraient s'étonner qu'ils ne soient pas plus nombreux. Même si cette maison offre un large éventail de publications pour répondre aux exigences de sa politique éditoriale, il convient que les ouvrages religieux occupent une place importante.

Les 387 ouvrages dits de littérature se répartissent entre les habituelles catégories de publications littéraires, soit les romans, la poésie, le théâtre et les essais, critiques, histoires littéraires. Il est opportun de mentionner que la plupart des grands écrivains québécois ont été publiés chez Fides. Qu'il suffise de mentionner, parmi tous ces auteurs, des écrivains aussi connus que Roger Brien, Jean Bruchési, Robert Choquette, Alfred Desrochers, Léo-Paul Desrosiers, François Hertel, Félix Leclerc, Frère MarieVictorin, Félix-Antoine Savard, Laure Conan, Louis Dantin, Octave Crémazie, Robert Elie, Jules Fournier, Louis Fréchette, Ringuet, Yves Thériault, Emile Nelligan et bien d'autres. 
Par ailleurs, il est particulièrement intéressant de constater que Fides a publié près de 300 ouvrages de littérature de jeunesse. Nées à la suite d'une grande enquête sur la lecture chez les jeunes, les Éditions Fides restent ainsi fidèles à une des principales raisons de leur fondation: fournir aux jeunes une littérature agréable, attirante, incitative, leur donner le goût de lire des bons livres, avec toutes les conséquences que l'habitude de la lecture peut entraîner sur le développement culturel, sur l'enrichissement du vocabulaire, sur. le perfectionnement de la langue, sur ce qu'on appelait à une certaine époque «la teste bien faicte». Les livres de cette catégorie couvrent la plupart des disciplines et pourraient se retrouver dans les autres catégories. Sauf qu'ils sont conçus pour les jeunes, aussi bien sur le plan rédactionnel que sur celui de la présentation graphique et artistique. Bien sûr, un grand nombre de ces livres de littérature de jeunesse sont des romans, mais on dénombre aussi, par exemple, des biographies, des ouvrages historiques et des ouvrages religieux adaptés aux jeunes.

C'est peut-être dans le secteur de l'historiographie canadienne que les Éditions Fides auront le plus mérité d'avoir rempli, pendant un bon moment, le rôle des presses universitaires existantes. Et le nombre de 194 ouvrages publiés dans cette discipline témoigne de leur importance. À peu près tous les grands historiens québécois de cette période ont publié chez Fides. On pense, par exemple, à Lionel Groulx, Guy Frégault, Marcel Trudel, Michel Brunet, Albert Faucher, Léon Gérin, Robert Rumilly, Robert-Lionel Séguin, Jean Hamelin, Fernand Ouellet et à d'autres que nous ne pouvons tous énumérer. Les oeuvres de ces historiens ont été déterminantes dans I'historiographie québécoise et leur rencontre chez un même éditeur témoigne de la confiance qu'ils lui accordaient et du rôle primordial et essentiel que cet éditeur a joué.

Les Éditions Fides ont publié 117 biographies durant ces cinquante années. Un certain nombre de ces ouvrages auraient pu appartenir à la catégorie précédente, tellement mince est quelquefois la cloison qui sépare la biographie et I'histoire. Et, bien sûr, les sujets de ces biographies logent dans toutes les disciplines, si bien que quelques biographies sont carrément des hagiographies et d'autres voisinent I'histoire littéraire. Les sujets de ces biographies vont de Hitler à Louis Riel, de Roosevelt à Sainte Odile, du Frère André [neuf biographies, qui s'en étonnerait?] à l'intendant Dupuy et à Pierre ElliotTrudeau.

C'est dans le secteur des sciences pures et appliquées que le nombre de publications est le plus faible, seulement 42 , soit en moyenne moins d'une publication par année. Cette faible activité ne serait-elle pas tout simplement le reflet d'une vie scientifique fort limitée durant la plus grande partie de cette période? Pourtant, même si la plupart des ouvrages scientifiques de ce secteur ne constituent pas des traités scientifiques de très haute rigueur, il semble qu'ils aient rendu beaucoup de services puisqu'ils ont connu une diffusion relativement importante. S'ils n'ont pas contribué à faire progresser les développements de la science, ils ont aidé à familiariser les lecteurs et, en particulier, les jeunes avec certaines disciplines scientifiques et, peut-être, à leur donner le goût d'en connaître davantage. On pense à la Géologie générale de Cailleux, à l'Initiation à la géologie de Laverdière et Morin [qui a connu 13 tirages], aux huit manuels d'Éléments de chimie de Réal Aubin.

Enfin, nous avons regroupé dans la dernière catégorie divers ouvrages que le cadre de ce travail ne nous permettait pas d'identifier avec plus de raffinement. Quelques ouvrages portent sur un aspect ou l'autre des beaux-arts comme, par exemple, le Dictionnaire du cinéma québécois de Michel Houle et Alain Julien ou le Paul-Emile Borduas de François-Marc Gagnon; d'autres ouvrages comme Le bibliothécariat du regretté Raymond Tanghe ou la Cuisine raisonnée, un classique par les religieuses de la Congrégation de Notre-Dame, ou encore Éditions et lecture par le Père Paul-Aimé Martin, Les fonds mutuels par Raphaël Pilon ou Les nations autochtones en Amérique du Nord par N'Tsuk, Robert Vachon et Yves Michaud. Voilà autant d'exemples parmi les 291 titres que nous avons regroupés dans cette section. Cet éclectisme lui-même nous rappelle donc que, pour promouvoir l'humanisme intégral, cet éditeur a accepté maints ouvrages dont la valeur intrinsèque est établie.

\section{Les collections}

Tous ces 1882 ouvrages sont presque tous intégrés dans l'une ou l'autre des 151 collections de cet éditeur, dont plusieurs sont particulièrement prestigieuses. Sans les énumérer toutes, il convient de mentionner, dans le secteur des livres religieux, les collections "Cahier de recherche éthique», "Foi et liberté», "Héritage et projet» qui, toutes trois, offrent des textes de recherche et de réflexion théologiques, souvent fort savants, tandis que la grande collection "L'Église aux quatre vents» publie, entre autres, des textes pontificaux dans un format et à un prix abordables. Dans le secteur littéraire, il faut d'abord citer la collection "Le Nénuphar», remarquable à maints égards. On l'a déjà comparée à la collection «La Pléiade» chez Gallimard. L'éditeur y publie les oeuvres qui offrent un caractère à la fois de permanence et de classicisme. Comme dans «La Pléiade», entrer 
dans le "Le Nénuphar» constitue une consécration. Et tous les lecteurs souhaitent que cette collection continue de s'enrichir de nouveaux textes qui répondent à ses critères d'inclusion.

D'autres collections littéraires ont également contribué à faire connaître des ouvrages québécois importants. Notons, entre autres, les collections "Alouette bleue», "Bibliothèque canadienne-française» et "Bibliothèque québécoise", "Écrivains canadiens d'aujourd'hui», "Le Goéland", "La grande aventure», "Rêve et vie». La collection "Classiques canadiens» mérite une place d'honneur, en offrant aux lecteurs un choix de 44 auteurs importants dont l'oeuvre intégrale est peu consultée, mais qui, grâce à ces petits ouvrages, ont la chance d'être au moins connus. Citons, à titre d'exemples, Samuel de Champlain, Pierre Biard, Elisabeth Bégon, Marc Lescarbot, Pierre Boucher, Frontenac, Laure Conan. Auteurs "classiques" de notre littérature et de notre histoire, leur oeuvre n'est souvent connue et consultée que par des spécialistes. Cette collection permet aux étudiants, par exemple, de s'initier à une oeuvre essentielle et à une période importante de notre histoire.
Enfin, dans le secteur historique, mentionnons trois collections: "Bibliothèque économique et sociale», "Histoire économique et sociale du Canada français» et surtout «Fleur de lys». Celleci est à l'histoire du Québec ce que la collection "Le Nénuphar» est à la littérature québécoise. Jusqu'à présent, onze historiens ont vu leur oeuvre figurer dans cette collection prestigieuse. Et encore une fois, il faut souhaiter que cette collection essentielle continue de s'enrichir

\section{Conclusion}

Cette maison d'édition poursuit donc depuis maintenant plus de cinquante années une oeuvre, au vrai sens de ce mot. Elle la poursuit dans la fidélité au mandat initial qu'elle s'était donné: celui d'offrir des ouvrages qui se situent dans la ligne de l'«humanisme intégral», qui assurent aux lecteurs ce "surplus d'âme» dont notre collectivité a tellement besoin. À cette oeuvre que les responsables des Éditions Fides ont placée à hauteur d'intelligence, notre collectivité québécoise doit une large part de son étonnante évolution

\section{wore SEAver \\ EXPRESS $M A G A Z I N E$ A CANADIAN Une divion de EMBSCRIPTION \\ Une division de E.M. Press inc. SERVICE}

- Spécialiste des revues françaises au Canada depuis 7 ans.

- Plus de 1000 titres de périodiques français, italiens, espagnols.

- Service personnalisé aux bibliotheques (information des éditeurs, réserve de numéros).

- Gestion informatisée des titres.

- Expédition des revues de Montréal

Une division de : E.M. Press $4001 \mathrm{~A}$, boulevard Robert.

Montréal, Québec

H1Z 4 H6 Canada

Tél. . (514) 374-9811

1-800-363-7141
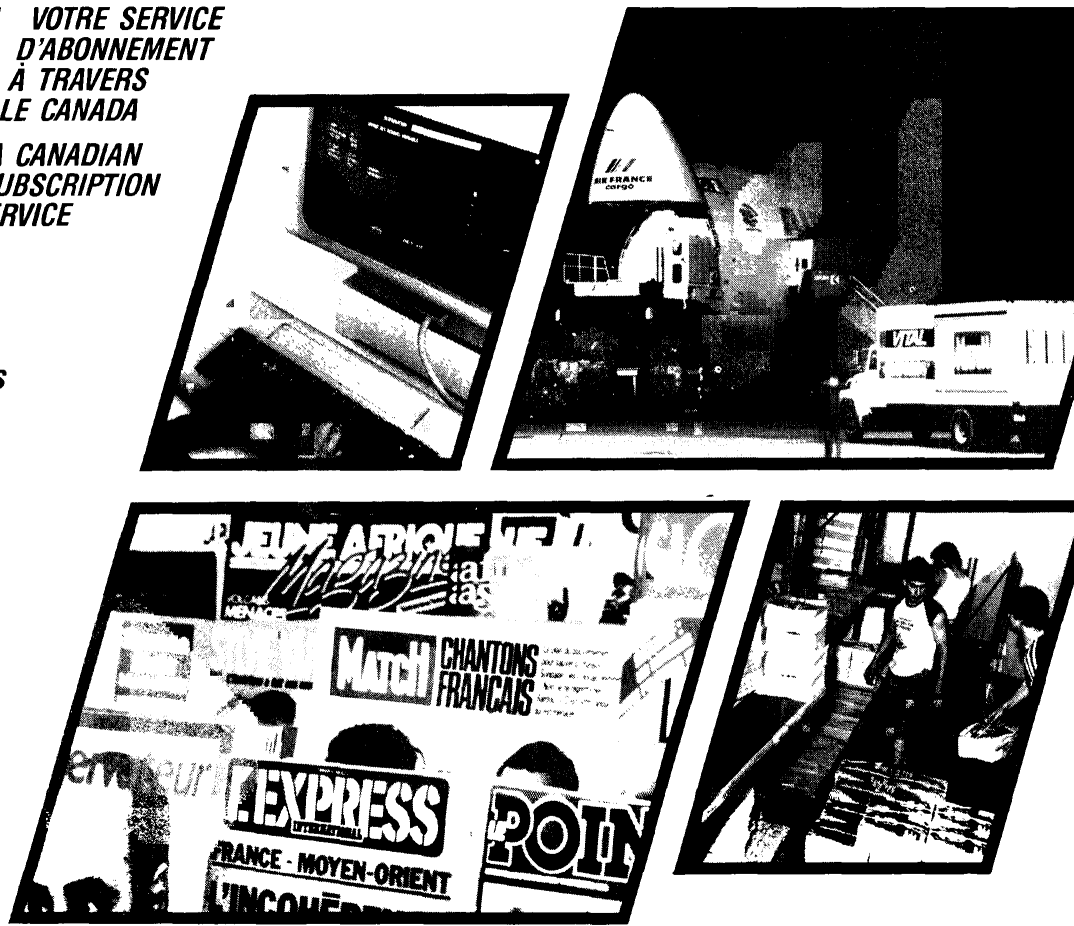